\title{
Association between benzodiazepine use and exacerbations and mortality in patients with asthma: A matched case-control and survival analysis using the United Kingdom Clinical Practice Research Datalink
}

\author{
Georgina Nakafero $^{1 *}$, Robert D. Sanders ${ }^{2}$, Jonathan S. Nguyen-Van-Tam ${ }^{1}$, Puja R. Myles \\ ${ }^{I}$ Division of Epidemiology and Public Health, University of Nottingham \\ ${ }^{2}$ Department of Anesthesiology, University of Wisconsin, Madison \\ *Corresponding author: Georgina Nakafero, Division of Epidemiology and Public Health, University of Nottingham, \\ Nottingham, NG $72 R D$ \\ E-mail: mcxgn@nottingham.ac.uk
}

\begin{abstract}
Purpose: To investigate the association between the GABAergic drugs, benzodiazepines or zopiclone, and the occurrence of asthma exacerbations and subsequent mortality in a cohort of asthma patients.

Methods: 105,747 patients without asthma exacerbation and 25,895 patients with exacerbated asthma were included. A nested case-control study probed the association between benzodiazepines or zopiclone and occurrence of asthma exacerbation (primary outcome) using conditional logistic regression. Cox regression was used to determine the association between the drugs and all-cause mortality in patients with recorded asthma exacerbation. Adjusted matched odds ratios (adj mOR), and adjusted hazard ratios (adj HR) with $95 \%$ confidence intervals $(\mathrm{CI})$ are presented.
\end{abstract}

Results: Current benzodiazepine use was associated with increased occurrence of asthma exacerbation (adj mOR 1.49; 1.15-1.93; $\mathrm{P}=0.001$ ) as was current zopiclone use (adj mOR 1.59; $95 \%$ CI 1.37-1.85; $\mathrm{P}<0.001$ ). In patients with an asthma exacerbation, current benzodiazepine use was associated with increased all-cause mortality during a median follow-up of 2 years (adj HR 2.78; 95\% CI 1.26-6.12; $\mathrm{P}=0.011$ ), and the association between zopiclone use and all-cause mortality showed borderline statistical significance (adj HR 1.58; 95\% CI 0.98-2.54; $\mathrm{P}=0.058$ ).

Conclusion: Benzodiazepines and zopiclone may increase the likelihood of asthma exacerbation and benzodiazepines may also increase the likelihood of mortality following exacerbation. These data suggest that caution should be exercised when prescribing benzodiazepines to patients with asthma.

Key words: benzodiazepines, zopiclone, asthma exacerbation, mortality.

\section{INTRODUCTION}

Asthma affects 235 million people globally ${ }^{1}$ with estimated mortality of 1.1 and 1.6 per 100 , 000 patients per year in the United States (US) ${ }^{2}$ and the United Kingdom (UK) ${ }^{3}$ respectively. One potentially important strategy to reduce this burden of disease is to identify modifiable 
risk factors, including medications that predispose to exacerbation of asthma and subsequent mortality.

Benzodiazepines are widely prescribed especially for the treatment of anxiety and insomnia, ${ }^{4}$ through modulation of inhibitory $\mathrm{V}$-amino butyric acid Type $\mathrm{A}\left(\mathrm{GABA}_{\mathrm{A}}\right)$ receptors in the brain. ${ }^{5}$ Occasionally they may also be used in treatment of severe anxiety that presents during asthma attacks. ${ }^{6}$ However they are not innocuous; accumulating evidence suggest they may cause harm $^{7,8}$, including increased mortality in the general population ${ }^{9}$ and risk of cancer. ${ }^{10}$ Benzodiazepines may also increase the risk from infection. ${ }^{11-13}$ Obiora et al found that benzodiazepine exposure in the community was associated with an increased risk of pneumonia and subsequent all-cause mortality following pneumonia. ${ }^{14}$ Meta-analysis of data from randomised controlled trials for non-benzodiazepine GABAergic hypnotics (zopiclone, ramelteon, zaleplon and zolpidem) also identified an increased risk of infection (risk ratio; 1.44, $95 \%$ confidence interval $1.25-1.64, \mathrm{p}<0.001) .{ }^{15}$ These data support evidence from critical care where benzodiazepine sedation is associated with increase in mortality in septic patients relative to non-GABAergic sedation. ${ }^{16}$ However, the data are somewhat inconsistent overall, with two studies ${ }^{17,18}$ suggesting a protective effect on pneumonia and some finding no statistical association. ${ }^{19-21}$ With regards to all-cause mortality, a systematic review conducted in 2009 found mixed and inconclusive results. ${ }^{22}$ Thus, further data are urgently required.

In asthma, increased expression of epithelial $\mathrm{GABA}_{\mathrm{A}}$ receptors leads to the overproduction of mucus. $^{23}$ Furthermore, allergen-induced asthma in mice increases the expression of benzodiazepine-sensitive $\mathrm{GABA}_{\mathrm{A}}$ receptors on alveolar macrophages. ${ }^{13}$ Augmented GABAergic signalling, such as through benzodiazepine treatment, impairs immune responses through intracellular acidosis of monocytes and macrophages, leading to increased mortality in animal models of infection ${ }^{13,24}$ supporting the observations in humans. ${ }^{9}{ }^{14}$ Given the 
intrinsic role of the pulmonary GABAergic system in the pathogenesis of asthma and accumulating data on potential harms of benzodiazepines especially from infections, we have evaluated GABAergic drugs in asthmatic patients in relation to the occurrence of asthma exacerbations, and mortality following asthma exacerbation.

\section{METHODS}

Data source

Data were extracted from the Clinical Practice Research Datalink (CPRD) that contains the medical records of over 13 million patients prospectively collected from over 600 general practices across the UK. CPRD has demonstrated a high level of completeness of clinical, diagnostic and prescription data according to validation studies. ${ }^{25}$ This electronic database is linked to other data sources for example hospital patient records (the Hospital Episode Statistics database [HES] and national death registry data (the Office of National Statistics [ONS]) which were utilised in this study (CPRD: http://www.cprd.com/).

\section{Sampling frame}

People with a diagnosis of asthma ( $n=225,543$ [Appendix 1]) were identified using relevant diagnostic codes (Read codes [Appendix3]) which map on to the international classification of disease (ICD-9) codes, for the study period 01/01/2005 through 31/12/2011.

\section{Outcome definition}

The primary outcome for the nested case-control analysis was physician diagnosed asthma exacerbation (cases), identified using Read codes. We used hospital data to identify missing patients using the ICD 10 code for status asthmaticus (J46)/or a record of hospitalisation occurring within 14 days of a primary care diagnosis of asthma exacerbation. The date of the first recorded diagnosis of asthma exacerbation during the study period was designated the index date. All asthma patients, in the study population, who had not experienced an 
exacerbation during the study period, were identified as potential controls. These were individually matched to cases by age at index date ( \pm 3 years), sex and general practice (matching on general practice i.e. primary care clinic, was performed to account for differences in prescribing policies and patient management followed in various primary care clinical settings). A maximum of six controls were matched to each case to give sufficient sample size for stratified analyses. The sample size was calculated using the Dupont Power and Sample size program (Appendix 2). ${ }^{26}$ The secondary outcome of interest was all-cause mortality (over a median follow-up time of 1.97 years) following a diagnosis of asthma exacerbation during the study period, investigated using survival analysis, including only those patients diagnosed with an asthma exacerbation (Appendix 1).

\section{Exposure definition}

Data were extracted for all recorded prescriptions of benzodiazepines and zopiclone. The most recent prescription prior to the asthma exacerbation diagnosis date (or the index date of the matched case for controls) was retained. Benzodiazepines were considered both as a class and as individual drugs. The three most frequently used individual benzodiazepines in the study population, namely, diazepam, temazepam and lorazepam were also identified for $a$ priori stratified analyses, representing long-acting, intermediate and short-acting agents respectively. Exposure was considered current when the most recent prescription was within the 30 days prior to the index date. The most commonly prescribed oral benzodiazepines in the UK typically have a 20-28 tablet pack-size. ${ }^{4}$ We used this to reason that long-term regular users of benzodiazepines would have repeat monthly prescriptions from their general practitioner. Presence of a repeat prescription defined long term drug use whereas the absence of it was considered short term use. Prescriptions in the period 31 to 90 days and >90 days before index date were classified as recent and past exposures respectively. No evidence of prescribing at any point in the patient's past primary care records defined 'never' users. 


\section{Potential confounders}

A validated weighted, comorbidity index (Charlson's comorbidity index) was adopted as a measure of comorbidity burden. ${ }^{27}$ In addition, we adjusted for depression, sleeping problems, psychosis, stress and anxiety, identified as important confounders in previous related studies 9 , ${ }^{14}$ but not covered by the Charlson index. Other potential confounders considered included age, sex, current smoking (most recent record), alcohol consumption (measured as units of alcohol consumed per week and categorised according to the UK weekly recommended alcohol consumption limits; <14units/week for women and <21units/week for men), socioeconomic status (measured using Index of Multiple deprivation (IMD) score quintiles) ${ }^{28}$ and body mass index (as recorded by general practitioners and categorised according to WHO guidelines). ${ }^{29}$ Current exposures (as defined previously) to statins, opioids, beta-2 agonists and topical respiratory corticosteroids (oral, inhaled, nasal and nebulised) were also considered.

\section{Statistical analyses}

Conditional logistic regression was used to investigate the association between drug exposure and asthma exacerbation. Cox regression modelling was used to assess the association between drug exposure and mortality following asthma exacerbation after checking that proportional hazards assumptions were met. Missing data were included in the analysis as dummy variables.

We constructed two multivariable models for each exposure variable: Model 1 included all covariates that were significantly associated with both asthma exacerbation and the exposure variable $(\mathrm{p}<0.05)$ and Model 2 included all potential confounders as listed in the previous section. We tested for interaction with comorbidities and where a statistically significant 
interaction was found the multivariable analysis was repeated, stratified by the interaction variable categories.

To quantify the proportion by which the incidence of asthma exacerbation among asthma patients would reduce if exposure to benzodiazepines was eliminated, ${ }^{30}$ the adjusted attributable fraction (AF) among the exposed and the adjusted population attributable fraction (PAF) were determined using the user-generated package 'punaf' in Stata. ${ }^{31,32}$ We also calculated the unadjusted number needed to harm (the inverse of the absolute risk increase). ${ }^{30}$ All statistical analyses were conducted in Stata version 12.

\section{RESULTS}

\section{Characteristics of study participants}

A total of 131,642 patients were included in the main analysis after excluding patients with anomalies in key dates e.g. registration and death dates (Appendix 1). Of these, 60,542(57.25\%) were females, 32,311(30.56\%) were aged below 18 years and $25,895(19.67 \%)$ had an asthma exacerbation of which nearly $8 \%(2,063)$ were severe. Benzodiazepine exposure was noted in 20,725 patients, comprising $15.74 \%$ of the study population overall. Patients with asthma exacerbation were more likely to be from more deprived settings, have a diagnosis of depression, be current smokers, overweight or obese and have had a prescription of corticosteroids, opioids and beta-2 agonists (Table 1).

Table 1: Characteristics of asthma patients with and without asthma exacerbation $(n=131,642)$

\begin{tabular}{|l|l|l|l|l|}
\hline \multicolumn{5}{|c|}{ Exacerbation of asthma } \\
\hline Characteristic & No $(\mathbf{n = 1 0 5 7 4 7}) \%$ & Yes $(\mathbf{n = 2 5 8 9 5 )} \%$ & Crude OR (95\% CI) & P Value \\
\hline Age (years) & & & & \\
\hline$<18$ & $32311(30.56)$ & $7945(30.68)$ & $\dagger$ & \\
\hline $18-44$ & $37089(35.07)$ & $8640(33.37)$ & & \\
\hline $45-65$ & $24595(23.26)$ & $6200(23.94)$ & & \\
\hline$>65$ & $11752(11.11)$ & $3110(12.01)$ & & \\
\hline Gender & & & & \\
\hline Male & $45205(42.75)$ & $10821(41.79)$ & $\dagger$ & \\
\hline Female & $60542(57.25)$ & $15074(58.21)$ & & \\
\hline IMD score quintiles & & & & \\
\hline
\end{tabular}




\begin{tabular}{|c|c|c|c|c|}
\hline 1 (least deprived) & 13898(13.14) & $2990(11.55)$ & 1 & \\
\hline 2 & 14001 (13.24) & $3478(13.43)$ & $1.18(1.11-1.26)$ & $\mathrm{P}$ trend $<0.001$ \\
\hline 3 & $12390(11.72)$ & $3018(11.65)$ & $1.18(1.10-1.26)$ & \\
\hline 4 & $12750(12.06)$ & $3219(12.43)$ & $1.25(1.16-1.33)$ & \\
\hline 5 (most deprived) & 11884(11.24) & $3014(11.64)$ & $1.29(1.20-1.39)$ & \\
\hline Missing & 40824(38.61) & $10176(39.30)$ & - & \\
\hline \multicolumn{5}{|l|}{ Current smoking } \\
\hline No & $63522(60.07)$ & $17260(66.65)$ & 1 & \\
\hline Yes & $16720(15.81)$ & $4753(18.35)$ & $1.12(1.08-1.17)$ & $<0.001$ \\
\hline Missing & $25505(24.12)$ & $3882(14.99)$ & - & \\
\hline \multicolumn{5}{|l|}{$\begin{array}{l}\text { Alcohol } \\
\text { consumption }\end{array}$} \\
\hline $\begin{array}{l}\leq \text { alcohol weekly } \\
\text { limit }\end{array}$ & $31262(29.56)$ & 8011 (30.94) & 1 & \\
\hline $\begin{array}{l}>\text { alcohol weekly } \\
\text { limit }\end{array}$ & $3953(3.74)$ & $943(3.64)$ & $0.93(0.86-1.00)$ & 0.059 \\
\hline Missing & \begin{tabular}{|l}
$70532(66.70)$ \\
\end{tabular} & $16941(65.42)$ & - & \\
\hline \multicolumn{5}{|l|}{ Anxiety } \\
\hline No & 104433(98.76) & $25446(98.27)$ & 1 & \\
\hline Yes & 1314(1.24) & $449(1.73)$ & $1.38(1.24-1.55)$ & $<0.001$ \\
\hline \multicolumn{5}{|l|}{ Stress } \\
\hline No & 105275(99.55) & $25734(99.38)$ & 1 & \\
\hline Yes & $472(0.45)$ & $161(0.62)$ & $1.40(1.16-1.68)$ & $<0.001$ \\
\hline \multicolumn{5}{|l|}{ Depression } \\
\hline No & $92720(87.68)$ & $21786(84.13)$ & 1 & \\
\hline Yes & 13027 (12.32) & $4109(15.87)$ & $1.34(1.29-1.40)$ & $<0.001$ \\
\hline \multicolumn{5}{|l|}{ Psychosis } \\
\hline No & 105418 (99.69) & $25788(99.59)$ & 1 & \\
\hline Yes & $329(0.31)$ & $107(0.41)$ & $1.28(1.02-1.61)$ & 0.030 \\
\hline \multicolumn{5}{|l|}{ Sleeping problems } \\
\hline No & 99123(93.74) & $23671(91.41)$ & 1.00 & \\
\hline Yes & $6624(6.26)$ & $2224(8.59)$ & $1.38(1.31-1.46)$ & $<0.001$ \\
\hline \multicolumn{5}{|l|}{$\begin{array}{l}\text { Charlson's } \\
\text { Comorbidity index } \\
\text { score }\end{array}$} \\
\hline 0 & 91472(86.50) & $21776(84.09)$ & 1 & \\
\hline $1-2$ & 11189(10.58) & $3286(12.69)$ & $1.20(1.15-1.26)$ & $\mathrm{P}$ trend $<0.001$ \\
\hline $3-5$ & $2710(2.56)$ & $773(2.99)$ & $1.13(1.03-1.24)$ & \\
\hline$>5$ & $376(0.36)$ & $60(0.23)$ & $0.60(0.45-0.79)$ & \\
\hline \multicolumn{5}{|l|}{ BMI } \\
\hline Normal weight & \begin{tabular}{|l|}
$24013(22.71)$ \\
\end{tabular} & 5733(22.14) & 1 & \\
\hline Underweight & 1679(1.59) & $410(1.58)$ & $0.94(0.84-1.06)$ & $\mathrm{P}$ trend $<0.001$ \\
\hline Overweight & 19429(18.37) & $5292(20.44)$ & $1.17(1.12-1.22)$ & \\
\hline Obesity & $16195(15.31)$ & $5306(20.49)$ & $1.41(1.35-1.47)$ & \\
\hline Missing & 44431(42.02) & 9154(35.35) & - & \\
\hline \multicolumn{5}{|c|}{$\begin{array}{l}\text { Corticosteroids } \\
\text { (nasal, oral, inhaled } \\
\text { and nebulised) }\end{array}$} \\
\hline No & \begin{tabular}{|l|}
$104024(98.37)$ \\
\end{tabular} & $23430(90.48)$ & 1 & \\
\hline Yes & $1723(1.63)$ & $2465(9.52)$ & $6.32(5.91-6.75)$ & $<0.001$ \\
\hline \multicolumn{5}{|l|}{ Opioids $^{\mathrm{TI}}$} \\
\hline No & 105394(99.67) & $25746(99.42)$ & 1 & \\
\hline Yes & 353(0.33) & $149(0.58)$ & $1.65(1.35-2.02)$ & $<0.001$ \\
\hline \multicolumn{5}{|l|}{ Beta-2-agonists ${ }^{\mathrm{dT}}$} \\
\hline No & \begin{tabular}{|l|}
$103468(97.8)$ \\
\end{tabular} & 23042(88.98) & 1 & \\
\hline Yes & $2279(2.16)$ & $2853(11.02)$ & $5.61(5.28-5.95)$ & $<0.001$ \\
\hline \multicolumn{5}{|l|}{ Statins $^{\text {बl }}$} \\
\hline No & 105321(99.60) & $25779(99.55)$ & 1 & \\
\hline
\end{tabular}




\begin{tabular}{|c|c|c|c|c|}
\hline Yes & $426(0.40)$ & $116(0.45)$ & $1.02(0.82-1.26)$ & 0.858 \\
\hline
\end{tabular}

\section{Benzodiazepines and occurrence of asthma exacerbation}

Table 2 shows results for the association between benzodiazepines and zopiclone and asthma exacerbation. After adjustment for all variables (Model 2), anytime exposure to benzodiazepines was statistically significantly associated with increased occurrence of asthma exacerbation (adj mOR 1.29; 95\% CI 1.23-1.35; P<0.001). When only current use of benzodiazepines was considered, the point estimate increased for occurrence of asthma exacerbation (adj mOR 1.49; 95\% CI 1.15-1.93; P=0.001). Similarly, current zopiclone use was associated with increased occurrence of asthma exacerbation (adj mOR 1.59; 95\% CI 1.37-1.85; $\mathrm{p}<0.001)$. On considering individual benzodiazepines, the occurrence of asthma exacerbation was elevated for current use of diazepam and temazepam. However, the association between current lorazepam and asthma exacerbation lacked statistical significance. Duration of benzodiazepine use did not alter the observed association with asthma exacerbation with both long- and short-term users at risk (Table 2). Statistically significant interactions were found with Charlson's comorbidity index score. Table 3 shows the stratified analysis results. The association between anytime benzodiazepine use and asthma exacerbation was stronger among asthma patients without comorbidities.

Using unadjusted risks, we estimated that over a one year follow-up period, the number needed to harm was 22 (95\% CI 12-109), indicating that for every 22 asthma patients prescribed benzodiazepines annually, one additional asthma exacerbation could be expected. Moreover, in asthma patients prescribed benzodiazepines (20,725 Patients), $4.2 \%$ of all asthma exacerbations were attributable to benzodiazepines (adjusted AF 4.23; 95\% CI 3.54, 4.92). An adjusted PAF of 3.39 (95\% CI 2.83, 3.94) suggests that $3.4 \%$ of asthma 
exacerbations in all asthma patients could be reduced if use of benzodiazepines was eliminated.

Table 2: Association of benzodiazepines/zopiclone exposure with asthma exacerbation

\begin{tabular}{|c|c|c|c|c|c|}
\hline \multirow[t]{2}{*}{ Exposure } & \multirow[t]{2}{*}{$\begin{array}{l}\begin{array}{l}\text { Controls } \\
(\mathrm{n}=105747) \%\end{array} \\
\end{array}$} & \multirow[t]{2}{*}{$\begin{array}{l}\text { Cases } \\
(\mathrm{n}=\mathbf{2 5 8 9 5}) \%\end{array}$} & \multirow[t]{2}{*}{ OR $(95 \% \text { CI })^{\dagger}$} & \multicolumn{2}{|c|}{ Adjusted models OR (95\% CI) } \\
\hline & & & & Model 1 & Model 2 \\
\hline \multicolumn{6}{|c|}{ Benzodiazepines } \\
\hline Never & $90156(85.26)$ & 20761(80.17) & 1.00 & 1.00 & 1.00 \\
\hline Ever & 15591(14.74) & 5134(19.83) & $1.46(1.40-1.52)$ & $1.26(1.21-1.31)$ & $1.29(1.23-1.35)$ \\
\hline \multicolumn{6}{|c|}{ BZD timing } \\
\hline Non- use & $90156(85.26)$ & 20761(80.17) & 1 & 1.00 & 1.00 \\
\hline Current & $238(0.23)$ & $97(0.37)$ & $1.78(1.39-2.27)$ & $1.51(1.18-1.94)$ & $1.49(1.15-1.93)$ \\
\hline Recent & $415(0.39)$ & $151(0.58)$ & $1.63(1.34-1.97)$ & $1.39(1.14-1.69)$ & $1.36(1.11-1.66)$ \\
\hline Past & $14938(14.13)$ & $4886(18.87)$ & $1.45(1.39-1.51)$ & $1.25(1.20-1.30)$ & $1.28(1.23-1.34)$ \\
\hline \multicolumn{6}{|c|}{ BZD duration } \\
\hline Non-use & $90156(85.26)$ & 20761(80.17) & 1.00 & 1.00 & 1.00 \\
\hline Short-term & $14453(13.67)$ & $4786(18.48)$ & $1.46(1.41-1.53)$ & $1.27(1.21-1.32)$ & $1.30(1.24-1.35)$ \\
\hline Long-term & $1138(1.08)$ & $348(1.34)$ & $1.37(1.21-1.56)$ & $1.15(1.01-1.31)$ & $1.17(1.02-1.33)$ \\
\hline \multicolumn{6}{|l|}{ Diazepam } \\
\hline Never & $95806(90.60)$ & $22479(86.81)$ & 1.00 & 1.00 & 1.00 \\
\hline Ever & $9941(9.40)$ & $3416(13.19)$ & $1.47(1.41-1.54)$ & 1.31(1.25-1.37) & $1.33(1.27-1.40)$ \\
\hline \multicolumn{6}{|c|}{ Diazepam timing $^{\ddagger}$} \\
\hline Current & $164(0.16)$ & $62(0.24)$ & $1.64(1.21-2.21)$ & 1.38(1.01-1.87) & $1.42(1.04-1.94)$ \\
\hline Recent & $289(0.27)$ & $107(0.41)$ & $1.60(1.27-2.01)$ & $1.39(1.10-1.76)$ & $1.35(1.06-1.71)$ \\
\hline Past & 9488(8.97) & $3247(12.54)$ & $1.47(1.40-1.53)$ & $1.30(1.24-1.36)$ & $1.33(1.27-1.40)$ \\
\hline \multicolumn{6}{|c|}{ Temazepam } \\
\hline Never & $101774(96.24)$ & $24666(95.25)$ & 1.00 & 1.00 & 1.00 \\
\hline Ever & $3973(3.76)$ & $1229(4.75)$ & $1.23(1.15-1.32)$ & $1.05(0.98-1.13)$ & $1.07(0.99-1.15)$ \\
\hline \multicolumn{6}{|c|}{$\begin{array}{l}\text { Temazepam } \\
\text { timing }\end{array}$} \\
\hline Current & $41(0.04)$ & $25(0.10)$ & $2.39(1.42-4.01)$ & $2.13(1.25-3.62)$ & $1.93(1.10-3.38)$ \\
\hline Recent & $87(0.08)$ & $28(0.11)$ & $1.28(0.82-2.00)$ & $1.09(0.69-1.70)$ & $1.10(0.70-1.74)$ \\
\hline Past & $3845(3.64)$ & $1176(4.54)$ & $1.22(1.14-1.31)$ & $1.04(0.96-1.12)$ & $1.06(0.98-1.14)$ \\
\hline \multicolumn{6}{|c|}{ Lorazepam } \\
\hline Never & 105339(99.61) & $25770(99.52)$ & 1.00 & 1.00 & 1.00 \\
\hline Ever & $408(0.39)$ & $125(0.48)$ & $1.26(1.03-1.55)$ & $1.08(0.88-1.34)$ & $1.11(0.90-1.38)$ \\
\hline \multicolumn{6}{|c|}{$\begin{array}{l}\text { Lorazepam } \\
\text { timing }\end{array}$} \\
\hline Current & $9(0.01)$ & $1(0.00)$ & $0.48(0.06-3.81)$ & $0.47(0.06-3.86)$ & $0.48(0.06-3.96)$ \\
\hline Recent & $15(0.01)$ & $8(0.03)$ & $2.33(0.97-5.60)$ & $1.97(0.82-4.74)$ & $2.29(0.96-5.47)$ \\
\hline Past & $384(0.36)$ & $116(0.45$ & $1.24(1.00-1.54)$ & $1.06(0.86-1.32)$ & $1.08(1.05-1.60)$ \\
\hline \multicolumn{6}{|l|}{ Zopiclone } \\
\hline Never & 99401(94.00) & $23662(91.38)$ & 1.00 & 1.00 & 1.00 \\
\hline Yes & $6346(6.00)$ & $2233(8.62)$ & $1.50(1.42-1.58)$ & $1.26(1.19-1.33)$ & $1.28(1.21-1.36)$ \\
\hline \multicolumn{6}{|l|}{ Zopiclone } \\
\hline Non- use & 99401(94.00) & 23662(91.38) & 1.00 & 1.00 & 1.00 \\
\hline Current & $645(0.61)$ & $295(1.14)$ & $1.91(1.65-2.20)$ & $1.57(1.35-1.82)$ & $1.59(1.37-1.85)$ \\
\hline Recent & $364(0.34)$ & $135(0.52)$ & $1.60(1.30-1.96)$ & $1.31(1.06-1.62)$ & $1.34(1.08-1.65)$ \\
\hline Past & $5337(5.05)$ & $1803(6.96)$ & $1.44(1.36-1.53)$ & $1.22(1.14-1.29)$ & $1.24(1.16-1.32)$ \\
\hline
\end{tabular}

Note: BZD refers to benzodiazepines; statistically significant results are highlighted in bold

${ }^{\ddagger}$ reference group is benzodiazepines never users; $\uparrow$ adjusted for age, sex and general practice (matching variables); Model 1 adjusted for age, sex, general practice, Charlson's comorbidity index score, sleep disorders, stress, anxiety, depression, 
psychosis, opioids, alcohol, current smoking, body mass index and multiple deprivation score; Model 2: Model 1 covariates, beta 2 agonists, statins and corticosteroids.

Table 3: Association of asthma benzodiazepines and asthma exacerbation stratified by Charlson's comorbidity score

\begin{tabular}{|l|l|l|l|l|}
\hline Exposure variable & \multicolumn{4}{|l|}{ Adjusted mOR ${ }^{\dagger}$ stratified by Charlson's comorbidity index (CCI) score } \\
\hline & CCI score 0 & CCI score 1-2 & CCI score 3-5 & CCI score >5 \\
\hline Benzodiazepines & & 1.00 & & \\
\hline No & 1.00 & $\mathbf{1 . 2 4}(1.08-1.43)$ & $1.04(0.72-1.49)$ & $\mathrm{x}$ \\
\hline Yes & $\mathbf{1 . 3 6}(\mathbf{1 . 2 9 - 1 . 4 3 )}$ & & & \\
\hline Benzodiazepines & & & & \\
\hline Current & $\mathbf{1 . 6 0}(\mathbf{1 . 1 8 - 2 . 1 9})$ & $0.63(0.25-1.55)$ & $\mathrm{x}$ & $\mathrm{x}$ \\
\hline Recent & $\mathbf{1 . 4 5 ( 1 . 1 3 - 1 . 8 5 )}$ & $1.68(0.82-3.41)$ & $\mathrm{x}$ & $\mathrm{x}$ \\
\hline Past & $\mathbf{1 . 3 5}(\mathbf{1 . 2 8 - 1 . 4 3 )}$ & $\mathbf{1 . 2 5}(\mathbf{1 . 0 8 - 1 . 4 4 )}$ & $\mathrm{x}$ & \\
\hline
\end{tabular}

Note: ${ }^{\sharp}$ Reference group is 'non benzodiazepine users'

†adjusted for age, sex, general practice, sleep disorders, stress, anxiety, depression, psychosis, opioids, beta 2 agonists and corticosteroids alcohol, current smoking, body mass index and multiple deprivation score statins

Statistically significant results are highlighted in bold

${ }^{\times}$could not be calculated due to insufficient data

\section{Benzodiazepines and mortality following asthma exacerbation}

Table 4 presents HRs for the association between benzodiazepines or zopiclone exposures and all-cause mortality subsequent to asthma exacerbation over the study period (median follow-up 1.97 years). Benzodiazepine use was found to be significantly associated with increased mortality following asthma exacerbation (adj HR 1.32; 95\% CI 1.08-1.61; $\mathrm{p}=0.006$ ) with current and recent prescriptions associated with the greatest effect. Individually, only diazepam was significantly associated with increased mortality over the study period (adj HR 1.27; $95 \%$ CI 1.01-1.59; $\mathrm{P}=0.037)$. However, the sample sizes were insufficient to fully investigate the individual effects of other benzodiazepines on mortality. The unadjusted number needed to harm (NNH) was estimated as 17(95\% CI 9 - 133); thus for every 17 asthma patients taking benzodiazepines, one additional death could be expected within a median follow-up time of 2 years. The association between current zopiclone use and all- 
cause mortality following asthma exacerbation showed borderline statistical significance (adj

HR 1.58; 95\% CI 0.98-2.54; P=0.058).

Table 4: Association between benzodiazepines/zopiclone use and all-cause mortality following asthma exacerbation in all ages $(n=25887)$

\begin{tabular}{|c|c|c|c|c|}
\hline \multirow[t]{2}{*}{ Drug } & \multirow{2}{*}{$\begin{array}{l}\text { Numbers } \\
\text { dead }(\%) \\
(n=459)\end{array}$} & \multirow[t]{2}{*}{$\begin{array}{l}\text { Unadjusted model } \\
\text { HR(95\% CI })\end{array}$} & \multicolumn{2}{|l|}{$\begin{array}{l}\text { Adjusted models } \\
\text { HR }^{1}(95 \% \mathrm{CI})\end{array}$} \\
\hline & & & Model 1 & Model 2 \\
\hline \multicolumn{5}{|c|}{ Benzodiazepines } \\
\hline Never & $272(1.31)$ & 1.00 & 1.00 & 1.00 \\
\hline Ever & $187(3.64)$ & $3.14(2.61-3.79)$ & $1.33(1.09-1.63)$ & 1.32(1.08-1.61) \\
\hline \multicolumn{5}{|c|}{ Benzodiazepines } \\
\hline Non-use & $246(1.31)$ & 1.00 & 1.00 & 1.00 \\
\hline Current & $6(7.22)$ & $6.21(2.93-13.16)$ & $2.69(1.24-5.82)$ & $2.78(1.26-6.12)$ \\
\hline Recent & $6(5.30)$ & $5.24(2.59-10.58)$ & $3.38(1.64-6.94)$ & $3.40(1.65-6.98)$ \\
\hline Past & $158(3.52)$ & $3.02(2.50-3.66)$ & 1.27(1.04-1.56) & $1.26(1.03-1.54)$ \\
\hline \multicolumn{5}{|c|}{ Diazepam } \\
\hline Never & $353(1.57)$ & 1.00 & 1.00 & 1.00 \\
\hline Ever & $106(3.10)$ & $2.25(1.81-2.80)$ & $1.28(1.02-1.60)$ & 1.27(1.01-1.59) \\
\hline \multicolumn{5}{|c|}{ Temazepam } \\
\hline Never & $403(1.63)$ & 1.00 & 1.00 & 1.00 \\
\hline Ever & $56(4.56)$ & $3.02(2.28-3.40)$ & $1.14(0.85-1.53)$ & $1.14(0.85-1.52)$ \\
\hline \multicolumn{5}{|c|}{ Lorazepam } \\
\hline Never & $453(1.76)$ & 1.00 & 1.00 & 1.00 \\
\hline Ever & $6(4.80)$ & $3.42(1.53-7.66)$ & $1.61(0.72-3.63)$ & $1.61(0.72-3.63)$ \\
\hline \multicolumn{5}{|c|}{ Zopiclone } \\
\hline Never & $385(1.63)$ & 1.00 & 1.00 & 1.00 \\
\hline Ever & $74(3.31)$ & $2.39(1.86-3.06)$ & $1.17(0.89-1.53)$ & $1.16(0.88-1.51)$ \\
\hline \multicolumn{5}{|c|}{ Zopiclone timing } \\
\hline Non-use & $385(1.63)$ & 1.00 & 1.00 & 1.00 \\
\hline Current & $19(6.44)$ & $4.58(2.89-7.27)$ & $1.60(1.00-2.57)$ & $1.58(0.98-2.54)$ \\
\hline Recent & $5(3.70)$ & $2.86(1.18-6.92)$ & $1.64(0.67-4.04)$ & $1.68(0.68-4.19)$ \\
\hline Past & $50(2.77)$ & $1.99(1.48-2.67)$ & $1.03(0.75-1.41)$ & $1.02(0.74-1.39)$ \\
\hline
\end{tabular}

-Model 1: Adjusted for age, gender, Charlson's comorbidity index score, sleep disorders, anxiety, stress, depression, psychosis, opioids, steroids, statins, beta2agonists, alcohol consumption, current smoking, body mass index and Index of Multiple Deprivation

-Model 2: Adjusted for all the factors in model 1 as well as steroids, statins and beta2agonists that failed to reach statistical significance $(\mathrm{p}<0.05)$ in univariate analysis for asthma exacerbation and benzodiazepines.

-Statistically significant results are highlighted in bold

\section{Sensitivity Analyses}

As benzodiazepines and other GABAergic drugs are commonly used as hypnotics and anxiolytics in adults, ${ }^{33,34}$ we conducted a sensitivity analysis restricted to study subjects aged 18 years and older. The results from this sensitivity analysis for the association of benzodiazepines and asthma exacerbation were similar to the original ones (sensitivity 
analysis results: adj mOR 1.28; 95\% CI 1.22, 1.33; P<0.001; original results: adj mOR 1.29; 95\% CI 1.23, 1.35; $\mathrm{P}<0.001)$.

To minimise bias from misclassification of asthma exacerbation, corticosteroid prescribing was considered a proxy indication of an asthma exacerbation. A sensitivity analysis excluding controls with a current steroid prescription, (1723[1.63\%]), yielded similar results to the original ones (sensitivity analysis results: adj mOR 1.29; $95 \%$ CI 1.23, $1.34 ; \mathrm{P}<0.001$, original results: adj mOR 1.29; 95\% CI 1.23, 1.35; $\mathrm{P}<0.001)$ ). In addition, an attempt was made to quantify missed exacerbations as determined from CPRD-HES linked data; $0.44 \%$ (287) of the exacerbations were recorded in HES but not in CPRD. A sensitivity analysis undertaken using only HES linked data (64,832 patients) yielded comparable results to the original ones (sensitivity analysis results: adj mOR 1.25; 95\% CI 1.18, 1.33; $\mathrm{P}<0.001$, original results: adj mOR 1.29; 95\% CI 1.23, 1.35; $\mathrm{P}<0.001)$.

Severity of disease may influence disease outcome including all-cause mortality. However, only $0.1 \%$ (128) of the sample had severe asthma. A subgroup analysis excluding these patients yielded no change in our results for the occurrence of asthma exacerbation (sensitivity analysis results: adj mOR 1.29; 95\% CI 1.23, 1.34; $\mathrm{P}<0.001$ original results: adj mOR 1.29; 95\% CI 1.23, 1.35; $\mathrm{P}<0.001)$. In addition, hospitalised exacerbations, considered as a proxy measure for severe asthma exacerbation in this study, were only found in 2063 (7.97\%) patients with asthma exacerbation. A subgroup analysis excluding these patients yielded comparable mortality results to original ones (subgroup analysis results: adj HR 1.40; 95\% CI 1.12, 1.75; $\mathrm{p}=0.001$, original results (adj HR 1.32; 95\% CI 1.08, 1.61; $\mathrm{p}=0.006)$.

\section{DISCUSSION}

Summary of main findings 
The findings of this study suggest that use of benzodiazepines and zopiclone is associated with increased occurrence of asthma exacerbation and 2-year mortality after exacerbation; a heightened likelihood of exacerbation associated with current use increases biological plausibility. The high prevalence of asthma as a disease and, of benzodiazepines exposure $(15.7 \%)$ in this representative cohort, suggests our findings have public health significance.

\section{Strengths and limitations}

To our knowledge, this is the first epidemiological study to examine benzodiazepine use and the occurrence of asthma exacerbation and subsequent mortality. Use of the CPRD database has enhanced the generalisability of these findings and also allowed adequately powered analyses. Data on drug exposures are collected prospectively, prior to asthma exacerbation, thus eliminating any recall bias. Moreover, reporting bias is virtually eliminated as data are routinely collected, prospectively by general practitioners during patient consultation for patient care and medico-legal record-keeping. Using cohort data eliminates any temporal biases relating to timing of exposure. A conditional logistic regression was performed with cases and controls individually matched on age, sex, and general practice and further adjustment for a wide range of potential confounders including Charlson's comorbidity index score $^{27}$ and other specific diseases identified as confounders of the relationship between benzodiazepines and disease outcomes (sleeping problems, anxiety, stress, psychosis and depression). ${ }^{9}{ }^{14}$ In addition, the effect of other drugs that could be concurrently prescribed with benzodiazepines (e.g. opioids) and other factors that may trigger an asthma exacerbation (e.g. smoking) were controlled for. The data on important confounders like smoking and alcohol are usually gathered in primary care, especially for patients presenting with alcoholrelated and smoking-related disease, in response to the introduction of incentive payments in 2004 by the Quality Outcomes Framework. ${ }^{35}$ However, the inherent limitations of 
observational studies using routine databases to deal with confounding should also be recognised, in particular residual and unmeasured confounders (for example nutritional and physical factors which are not routinely recorded in CPRD). Read codes were used to identify disease variables, therefore variations in coding practices in different general practices, could have led to misclassification of asthma exacerbation. This is likely to be a non-differential bias that if it occurred, would have shifted the effect size towards unity. To minimise misclassification bias, corticosteroid prescribing was considered a proxy indication of an asthma exacerbation however this sensitivity analysis did not affect the results. Care was also taken to avoid multiple counting of a single asthma exacerbation (i.e. multiple consultations for the same episode) by analysing only the first documented asthma exacerbation in the study period.

The use of prescription data does not allow any assessment of compliance with treatment. Whilst this could lead to misclassification of exposure, the effects are likely to be nondifferential and would therefore bias results towards unity. Nevertheless, the observed associations still remained when we assessed chronic benzodiazepine use as indicated by repeat prescriptions (Table 2).

Our calculations suggest that benzodiazepines may account for nearly $3.4 \%$ of exacerbations in asthma patients and that one additional asthma exacerbation would be triggered within a year of using benzodiazepines for every 22 asthma patients. In addition, our findings suggest that for every 17 asthma patients taking benzodiazepines, one additional death could be expected within a median follow-up time of 2 years. However, these results should be interpreted with caution as the NNH estimates are based on unadjusted risks; and while the PAF has been calculated using adjusted risk estimates, both measures assume a causal relationship that an observational study such as this cannot establish. Moreover, our study did 
not assess drug dosages. Future studies should look at drug dosages and frequency in greater detail.

\section{Comparison with existing literature}

It is not possible to make a direct comparison of the results from our study with earlier findings as ours is the first study we are aware of that directly assesses benzodiazepines effect in asthma patients. Recently a similar observation has been made for patients with chronic obstructive pulmonary disease where benzodiazepines increased outpatient exacerbations and visits to the emergency room. ${ }^{8}$ Obiora et al also found that benzodiazepines were associated with an increased risk of pneumonia and pneumonia-related mortality in a non-selected population. ${ }^{14}$ Results for both benzodiazepines as a class and individual benzodiazepines are in line with those from the current study. In both studies, the highest risks were observed in relation to current use of benzodiazepines, particularly diazepam. The diazepam finding may be attributable to its prolonged half-life and active metabolites. ${ }^{36}$ However we suspect that the non-significant association observed for other individual benzodiazepines with mortality could be due to relatively low usage in the population studied and that this is in fact a drug class effect.

It is also interesting to note that patients with a lower comorbidity burden were at increased risk of worse outcome from benzodiazepines similar to previous observations with pneumonia. ${ }^{14,37}$ We surmise that the presence of comorbidity reduces the proportion of risk attributable to benzodiazepine exposure in the individual patient.

Our observation that benzodiazepine, and zopiclone exposure, are associated with an increased odds of asthma exacerbation is biologically plausible given the role of $\mathrm{GABA}_{\mathrm{A}}$ 
receptor signalling in the pathogenesis of asthma ${ }^{23}$ and immunity in the lung. ${ }^{13}$ While epidemiological studies cannot prove a causal relationship between benzodiazepines and asthma exacerbations, a large well-conducted epidemiological study can detect adverse drug effect signals that may be missed in the average randomised controlled drug trial. ${ }^{38}$ Ideally, a large RCT should be conducted to investigate this association (for the outcome of 'exacerbations') ethical considerations permitting; alternatively, additional evidence from large prospective cohort studies, using standardised data collection protocols and selfcontrolled case series that would account for fixed confounding via intra-person comparisons, ${ }^{39}$ could strengthen the existing evidence.

\section{CONCLUSION}

Benzodiazepines are prescribed in up to one-sixth of patients with asthma. This study shows that these drugs along with zopiclone may increase the risk of asthma exacerbation; the risk of mortality after exacerbation may also be increased. Caution should be exercised when prescribing benzodiazepines in patients with asthma.

\section{Key points}

- A high prevalence of benzodiazepines use (15.7\%) was found in the asthma cohort studied.

- Having been prescribed a benzodiazepines within 30 days prior, was associated with an approximately $50 \%$ increased likelihood of asthma exacerbation.

- The non-benzodiazepine hypnotic, zopiclone, which also works via activation of $\mathrm{GABA}_{\mathrm{A}}$ receptors, was associated with a similar increase in the likelihood of asthma exacerbation.

- Both benzodiazepines and zopiclone were also found to be associated with increased mortality in the 2 years after an asthma exacerbation.

\section{ETHICS STATEMENT}


This study was approved by the independent ScientificAdvisory Committee for Medicines \& Healthcare products Regulatory Agency database research (ISAC Reference number: 11_098RA).

\section{ACKNOWLEDGEMENT}

This research was funded by the University of Nottingham Vice-Chancellor's Scholarship for Research Excellence scholarship award.

\section{AUTHORS' CONTRIBUTIONS}

Ms. Nakafero had full access to all the data in the study and takes responsibility for the integrity of the data and the accuracy of the data analysis. Ms. Nakafero performed the data management and analysis while all authors contributed to the study design, drafting of the article, reviewing for intellectual content and approval of the final version.

\section{REFERENCES}

1. The International Union Against Tuberculosis and Lung Disease. The Global Asthma Report 2011. Paris, France, 2011.

2. CDC. FastStats:Asthma. http://www.cdc.gov/nchs/fastats/asthma.htm (accessed 06/06/2014).

3. Royal College of Physicians. Why asthma still kills: the National Review of Asthma Deaths (NRAD) Confidential

Enquiry report. London: RCP, 2014.

4. BNF., editor. British National Formulary 63. London: BMJ Group and the Royal Pharmaceutical Society of Great Britain 2012.

5. Rudolph U, Crestani F, Benke D, et al. Benzodiazepine actions mediated by specific [gamma]aminobutyric acidA receptor subtypes. Nature 1999; 401(6755): 796-800.

6. Kil N, Zhu JF, VanWagnen C, Abdulhamid I. The effects of midazolam on pediatric patients with asthma. Pediatr Dent 2003; 25(2): 137-42.

7. Amarasuriya UK, Myles PR, Sanders RD. Long-term benzodiazepine use and mortality: are we doing the right studies? Current Drug Safety 2012; 7(5): 367-71.

8. Vozoris NT, Fischer HD, Wang X, et al. Benzodiazepine drug use and adverse respiratory outcomes among older adults with COPD. European Respiratory Journal 2014.

9. Weich $S$, Pearce HL, Croft $P$, et al. Effect of anxiolytic and hypnotic drug prescriptions on mortality hazards: retrospective cohort study. BMJ 2014; 348.

10. Kripke DF, Langer RD, Kline LE. Hypnotics' association with mortality or cancer: a matched cohort study. BMJ Open 2012; 2(1).

11. Laschi A, Descotes J, Tachon P, Evreux JC. Adverse influence of diazepam upon resistance to Klebsiella pneumoniae infection in mice. Toxicol Lett 1983; 16(3-4): 281-4.

12. Galdiero F, Bentivoglio C, Nuzzo I, et al. Effects of benzodiazepines on immunodeficiency and resistance in mice. Life Sciences; 57(26): 2413-23. 
13. Sanders RD, Godlee A, Goulding J, et al. Benzodiazepine augmented gamma-aminobutyric acid signaling increases mortality from pneumonia in mice. Crit Care Med, doi: 101097/CCMOb013e31827c0c8d 2013.

14. Obiora E, Hubbard R, Sanders RD, Myles PR. The impact of benzodiazepines on occurrence of pneumonia and mortality from pneumonia: a nested case-control and survival analysis in a population-based cohort. Thorax 2012.

15. Joya FL, Kripke DF, Loving RT, Dawson A, Kline LE. Meta-analyses of hypnotics and infections: Eszopiclone, ramelteon, zaleplon, and zolpidem. Journal of Clinical Sleep Medicine 2009; 5(4): 37783.

16. Pandharipande $P$, Sanders R, Girard T, et al. Effect of dexmedetomidine versus lorazepam on outcome in patients with sepsis: an a priori-designed analysis of the MENDS randomized controlled trial. Critical Care 2010; 14(2): R38.

17. Almirall J, Bolibar I, Balanzo X, Gonzalez CA. Risk factors for community-acquired pneumonia in adults: a population-based case-control study. European Respiratory Journal 1999; 13(2): 349-55.

18. Almirall J, Serra-Prat M, Baron F, Palomera E, Bolíbar I. The use of benzodiazepines could be a protective factor for community-acquired pneumonia (CAP) in $\leq 60$-year-old subjects. Thorax 2013.

19. Dublin S, Walker RL, Jackson ML, et al. Use of opioids or benzodiazepines and risk of pneumonia in older adults: a population-based case-control study. J Am Geriatr Soc 2011; 59(10): 1899-907.

20. Iqbal U, Syed-Abdul S, Nguyen PA, Jian W-S, Li Y-C. The impact of benzodiazepines on occurrence of pneumonia and mortality from pneumonia: a nested case-control and survival analysis in a population-based cohort. Thorax 2013; 68(6): 591-2.

21. Almirall J, Bolíbar I, Serra-Prat $\mathrm{M}$, et al. New evidence of risk factors for community-acquired pneumonia: a population-based study. European Respiratory Journal 2008; 31(6): 1274-84.

22. Charlson F, Degenhardt L, McLaren J, Hall W, Lynskey M. A systematic review of research examining benzodiazepine-related mortality. Pharmacoepidemiology \& Drug Safety; 18(2): 93-103.

23. Xiang $\mathrm{Y}-\mathrm{Y}$, Wang $\mathrm{S}$, Liu $\mathrm{M}$, et al. A GABAergic system in airway epithelium is essential for mucus overproduction in asthma. Nat Med 2007; 13(7): 862-7.

24. Sanders RD, Hussell T, Maze M. Sedation and Immunomodulation. Anesthesiol Clin 2011; 29(4): 687-706.

25. Herrett E, Thomas SL, Schoonen WM, Smeeth L, Hall AJ. Validation and validity of diagnoses in the General Practice Research Database: a systematic review. British Journal of Clinical Pharmacology 2010; 69(1): 4-14.

26. Dupont WD. Power calculations for matched case-control studies. Biometrics 1988; 44(4): 1157-68.

27. Charlson ME, Pompei P, Ales KL, MacKenzie CR. A new method of classifying prognostic comorbidity in longitudinal studies: development and validation. J Chronic Dis 1987; 40(5): 373-83.

28. Niggebrugge A, Haynes R, Jones A, Lovett A, Harvey I. The index of multiple deprivation 2000 access domain: a useful indicator for public health? Social Science \& Medicine 2005; 60(12): 2743-53. 29. World Health Organisation(WHO). BMI classification. 2013.

http://apps.who.int/bmi/index.jsp?introPage=intro 3.html.

30. Porta M, editor. A Dictionary of Epidemiology 5th ed: Oxford University press; 2008.

31. Newson RB. Scenario comparisons: How much good can we do? . 2012. http://www.imperial.ac.uk/nhli/r.newson/usergp/uk2012/newson ohp1.pdf.

32. Greenland S, Drescher K. Maximum likelihood estimation of the attributable fraction from logistic models. Biometrics 1993; 49(3): 865-72.

33. Woods JH, Katz JL, Winger G. Benzodiazepines: use, abuse, and consequences. Pharmacological Reviews 1992; 44(2): 151-347.

34. Gleason PP, Schulz R, Smith NL, et al. Correlates and prevalence of benzodiazepine use in community-dwelling elderly. J Gen Intern Med 1998; 13(4): 243-50. 
35. Doran T, Kontopantelis E, Reeves D, Sutton M, Ryan AM. Setting performance targets in pay for performance programmes: what can we learn from QOF? BMJ 2014; 348.

36. Rang H.P., Dale M.M., Ritter J.M., Flower R.J., G. H, editors. Rang and Dale's Pharmacology. seventh ed: Elsevier Inc.; 2012.

37. van de Nadort C, Smeets HM, Bont J, Zuithoff NPA, Hak E, Verheij TJM. Prognosis of primary care patients aged 80 years and older with lower respiratory tract infection. British Journal of General Practice 2009; 59(561): e110-e5.

38. Vandenbroucke JP. When are observational studies as credible as randomised trials? Lancet 2004; 363(9422): 1728-31.

39. Grosso A, Douglas I, MacAllister R, Petersen I, Smeeth L, Hingorani AD. Use of the selfcontrolled case series method in drug safety assessment. Expert opinion on drug safety 2011; 10(3): 337-40.

40. Myles PR, Hubbard RB, Gibson JE, Pogson Z, Smith CJ, McKeever TM. The impact of statins, ACE inhibitors and gastric acid suppressants on pneumonia mortality in a UK general practice population cohort. Pharmacoepidemiol Drug Saf 2009; 18(8): 697-703. 


\section{Appendices}

\section{Appendix 1 Asthma study subject selection procedure}

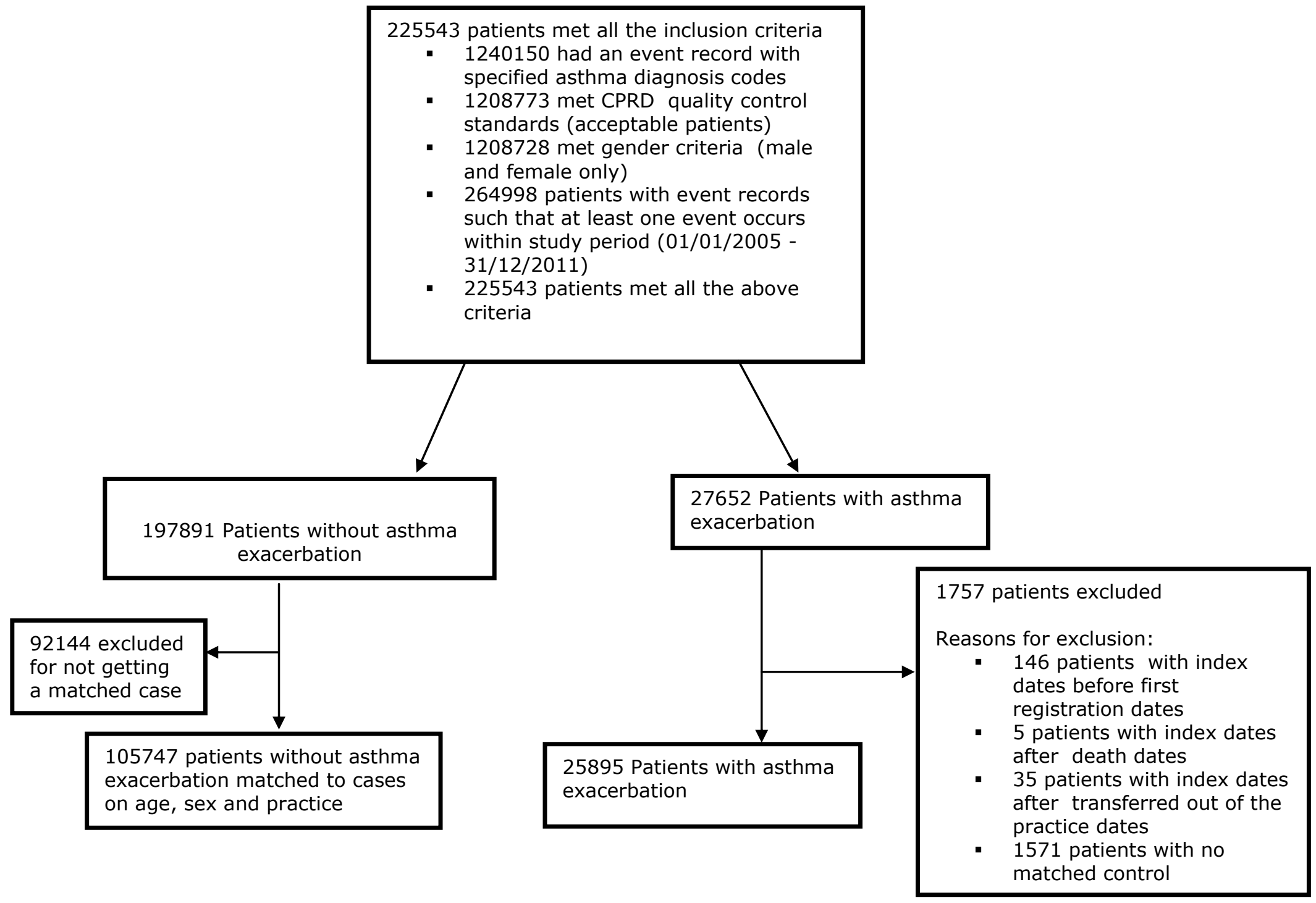




\section{Appendix 1 continued}

105747 patients without asthma exacerbation matched to cases

on age, sex and practice
131642 patients included in the logistic regression model to

investigate the association between

benzodiazepines/zopiclone and

asthma exacerbation
25895 Patients with asthma

exacerbation

\section{7 patients included in the}

Cox regression model to

investigate the association

between BZPs/zopiclone and

mortality subsequent to

exacerbation of asthma

asthma:

- 287 exacerbation in only HES

- 11979

exacerbations in only CPRD

- 1867

exacerbations

in both CPRD

and HES

2063 Patients had an

exacerbation of asthma

requiring hospitalisation

which was used as a proxy

measure for severe asthma

exacerbation
8 patients excluded

Reasons for exclusion:

- 2 patients with death dates on the same date as they transferred out of the practice

- 5 patients with death dates on last date of data collection at the practice

- 1 patient with death date before asthma exacerbation date

Over all total deaths:

- 282 deaths identified from ONS data

- 239 deaths in both

CPRD and ONS data

- 43 deaths in only ONS data

- 416 deaths identified
64832 patients had HES-CPRD

linked data utilised in sensitivity

analyses to cater for missed

exacerbations while using data

from only CPRD 
Appendix 2: Sample size calculation

1. Nested case-control study

\begin{tabular}{|l|l|l|l|l|l|}
\hline \multicolumn{2}{|l|}{ Outcome measure } & $\begin{array}{l}\text { Probability of } \\
\text { benzodiazepine } \\
\text { use in controls }\end{array}$ & $\begin{array}{l}\text { Odds ratio } \\
\text { (cases v. } \\
\text { Controls) }\end{array}$ & $\begin{array}{l}\text { Cases } \\
\text { needed }\end{array}$ & $\begin{array}{l}\text { Controls } \\
\text { needed }\end{array}$ \\
\hline $\begin{array}{l}\text { Outcome } \\
\text { measure }\end{array}$ & $\begin{array}{l}\text { Asthma } \\
\text { exacerbation }\end{array}$ & $0.17^{1}$ & $2.00^{2}$ & 119 & 714 \\
\cline { 2 - 7 } & $\begin{array}{l}\text { Asthma } \\
\text { exacerbations } \\
\text { requiring } \\
\text { hospitalisation }\end{array}$ & 0.17 & 1.50 & 378 & 2268 \\
\hline 2. Cohort study & $\begin{array}{l}\text { Ratio } \\
\text { unexposed to } \\
\text { exposed }\end{array}$ & $\begin{array}{l}\text { Hazard ratio } \\
\text { (unexposed } \\
\text { v. Exposed) }\end{array}$ & Exposed & patients & Unexposed \\
& & 3 & 1.2 & 718 & 2154 \\
\hline Outcome measure & mortality & 3 & & \\
\hline
\end{tabular}

a. Calculated using the Dupont Power and Sample size program as described in Dupont WD, Plummer WD: "Power and Sample Size Calculations: A Review and Computer Program", Controlled Clinical Trials 1990; 11:116-28.

b. Standard assumptions:

Powered at $80 \%$ with a type 1 error probability of 0.05, ratio of control to cases $6: 1$ (case-control analysis)

Correlation coefficient for exposure between matched cases and controls assumed to be 0.2 based on recommendations in Dupont $(1988)^{26}$

Median survival time in unexposed cases assumed to be 6 years (based on previous work in this topic area) with the ratio of unexposed to exposed subjects $3: 1^{40}$

${ }^{I}$ Probability of benzodiazepine use in controls based on population prevalence derived from The Health Improvement Network (THIN), a primary care database

${ }^{2}$ Based on previous point estimates obtained when investigating the association between benzodiazepine use and pneumonia incidence in THIN

Appendix 3: Readcodes for asthma

Asthma

$\underline{\text { Readcode } \quad \underline{\text { Read term }}}$

H33..00 Asthma

H33zz00 Asthma NOS 
H33..11 Bronchial asthma

H33z.00 Asthma unspecified

H330.00 Extrinsic (atopic) asthma

H330.12 Childhood asthma

H331.00 Intrinsic asthma

H331.11 Late onset asthma

$\mathrm{H} 33 \mathrm{z} 200 \quad$ Late-onset asthma

H330000 Extrinsic asthma without status asthmaticus

H332.00 Mixed asthma

H334.00 Brittle asthma

$\mathrm{H} 331 \mathrm{z00}$ Intrinsic asthma NOS

H331000 Intrinsic asthma without status asthmaticus

Exacerbation of asthma

$\underline{\text { Readcode }} \underline{\text { Read term }}$

H333.00 Acute exacerbation of asthma

H33z100 Asthma attack

H33z011 Severe asthma attack

H33z000 Status asthmaticus NOS

H330111 Extrinsic asthma with asthma attack

H33z111 Asthma attack NOS

H330100 Extrinsic asthma with status asthmaticus

H331111 Intrinsic asthma with asthma attack

H331100 $\begin{array}{ll}\text { Intrinsic } \\ \text { sthmaticus }\end{array}$

Asthma exacerbation requiring hospitalisation

$\underline{\text { Readcodes }} \underline{\text { Read term }}$

8H2P.00 Emergency admission, asthma

663m.00 Asthma accident and emergency attendance since last 、

663d.00 Emergency asthma admission since last appointment 